\title{
To Classify Spontaneous Motion Intention of Step Size by Using Cerebral Hemoglobin Information
}

\author{
Kai Zhu ${ }^{1}$, Chun-Guang Li², Hedian $\mathrm{Jin}^{3}$, Juan $\mathrm{Li}^{4}$, Haiyan $\mathrm{Hu}^{5}$ and Lining Sun ${ }^{6}$, Wei $\mathrm{Qu}^{7}$ \\ ${ }^{2}$ Key Laboratory of Robotics and System of Jiangsu Province, School of Mechanical and Electric Engineering, Soochow University, \\ Suzhou, China \\ 7 ECOVACS Robotics (Suzhou) Co. Ltd, China \\ ${ }^{2}$ lichunguang@suda.edu.cn, ${ }^{7}$ quw0219@foxmail.com
}

\begin{abstract}
To improve the effect of walking-assistive devices, there is a need for it to develop devices controlled by spontaneous intention of patients. In recent study, we identified spontaneous motion intention of walking step based on cerebral hemoglobin information. Twenty healthy subjects performed walking tasks in three levels of step size (small, normal and large). According to distribution features of signals' power spectral-density, six frequency bands $(0-0.18 \mathrm{~Hz}$ with an interval of $0.03 \mathrm{~Hz}$ for each band width) divided by applying wavelet packets decomposition were mainly analyzed. Feature vectors were extracted from the difference between oxygenated hemoglobin (oxyHb) and deoxygenated hemoglobin (dexoyHb) in different measuring channels in the six frequency bands. Support vector machine (SVM) method was utilized to classify the three levels of step sizes. Mean recognition accuracy achieved up to $83.3 \%$. The result indicated that it is possible to identify spontaneous walking by using cerebral hemoglobin information. This is helpful for enhancing the intelligence of walking-assistive devices and motivating the active control of patients, which further is profitable for enhancing self-confidence of patients.
\end{abstract}

\section{Introduction}

A variety of accidents, injuries and diseases make many people disabled. And the aging of population results in many people unable to walk. So it is necessary and meaningful to develop walking-assistive devices for the elderly or disabled people. At the same time, walkingassistive devices have been becoming more and more intelligent with the development of measuring technique and pattern recognition. What we need is not a robot which has flexible commands controlled by buttons, voice and so on. Assistive robots are further expected to achieve different functions based on the spontaneous intention of patients.

Among the various brain-signal acquisition methods (fNIRS[1, 2], EEG [3, 4], MRI [5, 6], EMG [7], etc.), functional near-infrared spectroscopy (fNIRS) is portable, quiet, accurate and not too sensitive to the motion of objects. fNIRS measures oxygenated hemoglobin (oxyHb) and deoxygenated hemoglobin (dexоyHb) concentration changes by shining nearinfrared light into the head (with three wavelengths of $780 \mathrm{~nm}, 805 \mathrm{~nm}$, and $830 \mathrm{~nm}$ ) and measuring the diffusively reflected light changes that originate from physiological changes in the skin and cortex [8]. Compared to EEG and MEG, fNIRS technology has a better spatial resolution. So it guarantees the accuracy of different measuring channels. At the same time, fNIRS technology has a better time sampling rate than MRI. Therefore, it can detect complete physiological signal, which is helpful for removing noise interference [9]. In addition, it can also realize real-time measurement. Currently, fNIRS technology has been widely used in disease monitoring [10], cognitive research [11], and brain activity imaging $[12,13]$. Previous studies have also explored the functional neural correlates of gait during dynamic tasks [14]. In sum, fNIRS is suitable for the study of identifying walking step. However, regions were often used to identify step size in previous studies [15]. At the same time, the motion of walking was mostly unnatural and not controlled by spontaneous intention [16]. These factors were not advantageous for the study of step size.

Our project proposed a method to identify walking step by using fNIRS technology. All the motions during the experiments were controlled by spontaneous intention. In the present work, the fNIRS signals in 22 channels were studied to seek for appropriate features. Wavelet packets decomposition and SVM were used to identify different step size. It is expected that the identified walking step could be applied in controlling walking-assistive devices. 


\section{Material and Methods}

\subsection{Subjects}

A total of twenty healthy subjects $(21 \pm 1$ years old, 17 male and 3 female) participated in our experiment. None of them had a history of any psychiatric or neurological disorder. The experimental procedure was explained to all of them in detail before they provided their verbal informed consent.

\subsection{Signal Acquisition}

\subsubsection{Measuring Instrument}

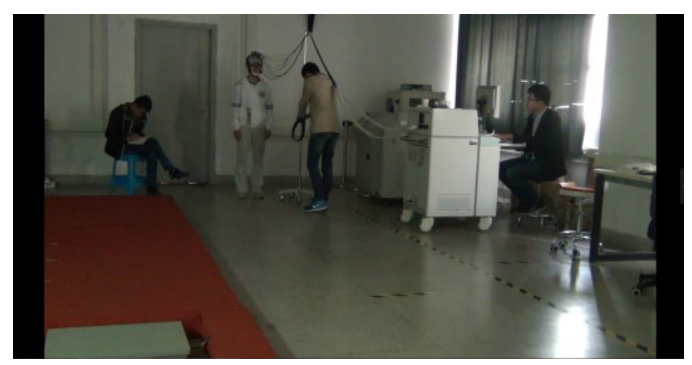

Figureure.1 Experimental diagram

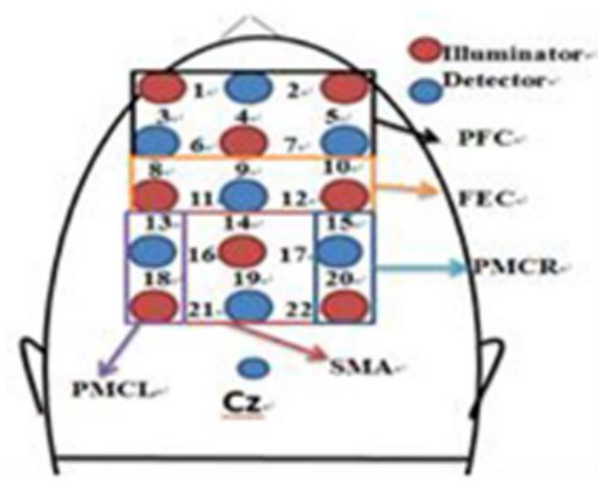

Figure.2 Diagram of measurement channels

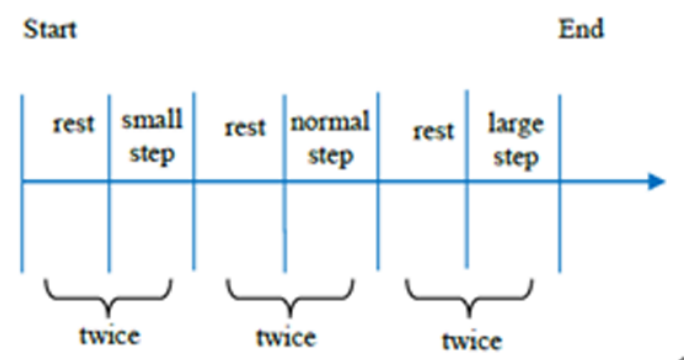

Figure. 3 The procedure of first group's experiment. The sequence of the first group was small, normal and large step size, while the sequence of the second group was large, normal and small step size.
In this study, a FORIE-3000 optical topography system (Shimadzu Corporation, Kyoto, Japan) [17] was used to measure concentration changes of oxygenated hemoglobin, deoxygenated hemoglobin, and total hemoglobin (totalHb) (Figure. 1). The raw fNIRS signals were acquired at a sampling period of $0.13 \mathrm{~s}$.

\subsubsection{Optode Arrangement}

One set of $3 \times 5$ parietal flash holder was applied to fix emitters and detectors. The arrangement of optodes are shown in the Figure. 2. The number of measurement channels was 22 . The measured regions included prefrontal cortex(PFCL: channels 1, 3, 4, 6 in the left hemisphere; PFCR: channels 2, 4, 5, 7 in the right hemisphere), premotor cortices(PMCL: channels 13, 18 in the left hemisphere; PMCR: channels 15, 20 in the right hemisphere), supplementary motor areas(SMAL: channels 14, 16, 19, 21 in the left hemisphere; SMAR: channels 14, 17, 19, 22 in the right hemisphere), frontal eye field (FECL: channels 8, 9, 11 in the left hemisphere; FECR: channels 9, 10, 12 in the right hemisphere). These areas have been confirmed to play an important role on regulating of walking [18].

\subsection{Experimental Procedure}

To eliminate the effects of experimental sequence, twenty participants were classified into two groups. The two groups were asked to perform experiments in a reverse motion sequence. The first group performed the walking tasks with a small, normal and large step size, while the second group performed the task with large, normal and small step size. In order to avoid the influence of dandruff, all the subjects were asked to wash hair one day before the experiment. During the experiment, all the motions were controlled by the subjects themselves without any implication or stimulation. That is, the start and the period of each task, as well as the period of each rest were all controlled by themselves. Cerebral hemoglobin information corresponding with spontaneous intention is preferable for enhancing application value of identification results.

The detailed procedure is shown in Figure. 3. Subjects were asked to walk in a fixed distance. The rest time was about 30 s and each level of step size was repeated twice. The marks of the start and end of one task were signed by an experiment operator. Before the experiment, all the tasks and rest were performed twice by the subjects to acquaint themselves with the tasks. During the experiment, they were asked to rest enough without any counting.

\section{Results}

In the first time of walking, there was some kind of nervous for the most subjects. Therefore, only the tasks performed in the second time were analyzed. 18 subjects ( 9 subjects of the first group and 9 subjects of the second group) were used to select features. And the two other subjects were used to verify. In our study, the difference 
between oxyHb and deoxyHb was mainly analyzed as the following steps.

\subsection{Data Preprocessing}

\subsubsection{Power Spectrum Density}

As the frequency band showed, the main power was distributed below $0.18 \mathrm{~Hz}$. So the raw signals were firstly low-pass filtered at $0.18 \mathrm{~Hz}$ to eliminate the effects of all kinds of noises. To confirm the correct division of wavelet packets decomposition, we analyzed the peak and valley values of power spectrum density. As shown in Figure. 4, the interval between the peak and valley values was around $0.03 \mathrm{~Hz}$. Therefore, as for the following wavelet packets decomposition, frequency band was divided into six parts $(0-0.03 \mathrm{~Hz}, 0.03-0.06 \mathrm{~Hz}$, $0.06 \mathrm{~Hz}-0.09 \mathrm{~Hz}, 0.09-0.12 \mathrm{~Hz}, 0.12 \mathrm{~Hz}-0.15 \mathrm{~Hz}, 0.15 \mathrm{~Hz}-$ $0.18 \mathrm{~Hz})$.

\subsubsection{Wavelet Packets Decomposition}

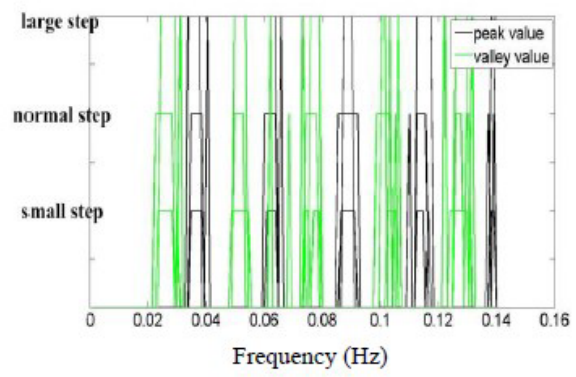

Figure.4 The peak values and valley values of power spectrum density. The green lines represent the peak values and the black lines denote the valley values. Frequency ranges of the peak or valley value observed from different subjects are shown on the vertical axis. And the three levels of walking step are shown on the horizontal axis.

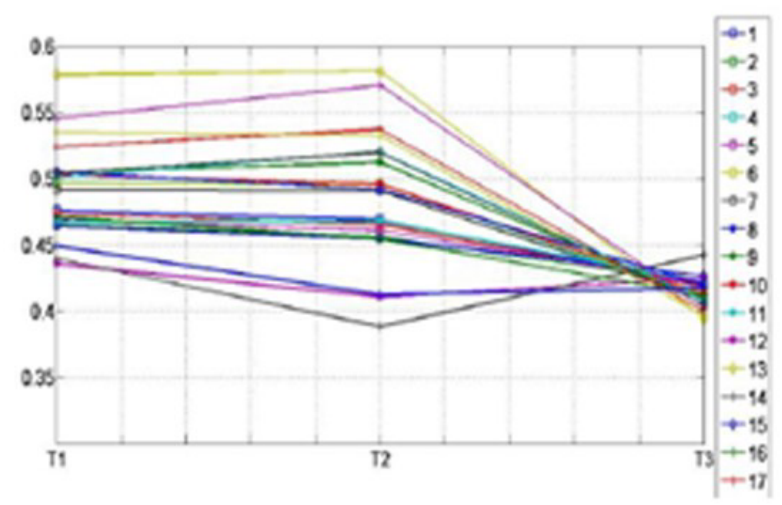

Figure. 5 Line chart of different channels in the three time periods. CWP: Coefficient of wavelet packet reconstruction. T1: The means of the 8 points before the start marks. T2: The means of the 8 points after the start marks. T3: The means of the 9 th-16th points after the start marks. Different colors represented 22 channels.

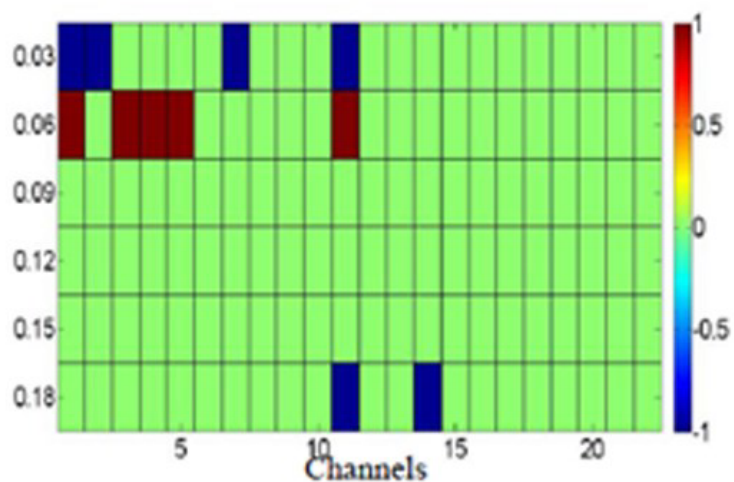

Figure.6 The results of statistical analysis. The red regions represent the values in the channels and frequency bands belonging to the top $30 \%$, and the blue regions represent the bottom $30 \%$.

First, 300 sampling points were truncated (180 sampling points before the start marks and 120 sampling points after the start marks) for the tasks in each step size. Second, the 300 sampling points subtracted their average value so as to reduce the effect of direct component. Third, the data was decomposed and reconstructed by 7-dimension wavelet packet algorithm [19-22]. If so, each frequency band was $0.03 \mathrm{~Hz}$ corresponding with the result of power spectrum density.

\subsection{Feature Extraction}

24 sampling points which show the best information of movement were further selected. The means of the 8 points before the start marks were recorded as $\mathrm{T} 1$; the means of the 8 points after the start marks were recorded as T2 and the means of the 9th-16th points after the start marks were recorded as T3. After that, means of the subjects calculated for T1, T2, and T3. In order to reduce the influence of individual difference in the two groups, the averages of the two groups were calculated respectively. Then, common features were selected based on the results of the two groups.

As for the measured 22 channels, each channel has three mean values of T1, T2, and T3 in each frequency band, and there were six frequency bands. Analyses were performed in both the time domain and frequency domain. As for the time domain, signal variation following as time (T1, T2, and T3) was observed for six frequency band. One of six frequency bands was taken for example in Figure. 5. If the values in one channel were distinctly larger or smaller than those in the other channels, or the values in one channel were obviously changed, the channel was selected as an important one. As for the frequency domain, information difference among the frequency bands was observed for each time period (T1, T2 and T3). It was the most obvious in T3.

To guarantee the correctness and universality of feature vectors, statistics method was applied to seek for important channels and frequency bands. For the values in the 22 channels in the 6 frequency bands, if the value in some channel and frequency band was the top or bottom 30 percent, and the same conclusion was 
obtained for $78 \%$ (14 subjects) of the 18 subjects, the corresponding channel in that frequency band was selected as one feature vector. One level of step size was taken as an example in Figure. 6.

\subsection{Identification of Step Size}

Since the features were much more obvious in $\mathrm{T} 3$ than those in $\mathrm{T} 1$ and $\mathrm{T} 2$. Thereby, the features in $\mathrm{T} 3$ were applied. Combining the features in the time domain and frequency domain, all the feature vectors were listed in Table 1. Finally these feature vectors were used to classify three levels of walking step by SVM [23]. Most features were concentrated in the regions of PMC and SMA. It suggested that the regions of SMA and PMC had a huge impact on controlling step size.

For SVM, among the 18 subjects whose data were used to extract features, 14 subjects' data were adopted as training set. Two subjects whose features were not obvious were removed. The data of the rest two subjects and two other subjects who were not used for feature extraction were used to verify the algorithm. Each subject performed three levels of step size. A classification accuracy of $83.3 \%$ was achieved.

Tabel 1. Features Vectors Used For Identifying Step Size

\begin{tabular}{|c|c|}
\hline Frequency & Channels(T3) \\
\hline $0-0.03 \mathrm{~Hz}$ & 16 \\
\hline $0.03-0.06 \mathrm{~Hz}$ & 12,13 \\
\hline $0.06-0.09 \mathrm{~Hz}$ & 1,11 \\
\hline $0.09-0.12 \mathrm{~Hz}$ & 19 \\
\hline $0.12-0.15 \mathrm{~Hz}$ & $8,14,15,17,18$ \\
\hline $0.15-0.18 \mathrm{~Hz}$ & $17,18,20,21$ \\
\hline
\end{tabular}

\section{Conclusion}

The present study demonstrated a new method to classify three levels of walking step, which was controlled by the subjects' spontaneous intention. And the classification accuracy of $83.3 \%$ was achieved. Spontaneous motion intentions are important in the control of walking-assistive devices. The proposed method had brighter prospects for realizing the intelligent control of walking assistive robots.

However, our study still had some limitations to solve before being applied in actual. First, the feature vectors were obtained in the time period of T3. Though the highest classification accuracy was achieved in T3, it resulted in a time delay of about $2 \mathrm{~s}$ for classification. Much more appropriate features should be found before the start of movement. In the following researches, we will try to apply the rate of cerebral hemoglobin change as features to eliminate the delay. The improvements of wavelet algorithm which are suitable for real-time application would also be considered. Further experiments and analyses would be performed on more subjects.

Moreover, current data was obtained from healthy subjects rather than paraplegics. However, our study is designed to be applied in paraplegics. Though their brains are normal, more and more patients would be analyzed in the near future.

\section{Acknowledgment}

This study was supported by the grants from the national high technology research and development program, China (863 Program: 2015AA042301) and Postdoctoral Science Foundation (2015M580461).

\section{References}

[1] M. Cope, "Methods of Quantitating Cerebral Near Infrared Spectroscopy Data." Advances in Experimental Medicine \& Biology, vol. 222, pp.183-189, 1988.

[2] F.F. Jobsis, "Noninvasive, infrared monitoring of cerebral and myocardial oxygen sufficiency and circulatory parameters." Science, vol. 198, no. 4323, pp.1264-1267, 1977.

[3] Giuseppe, Lisi, and M. Jun. "EEG Single-Trial Detection of Gait Speed Changes during Treadmill Walk.” PLOS One, 2015, 10(4): e0125479.

[4] Joseph T Gwin* and Daniel P Ferris. "An EEGbased study of discrete isometric and isotonic human lower limb muscle contractions." Journal of neuroengineering and rehabilitation, 2012, 9:35.

[5] Agnew, A. John, T. A. Zeffiro, and G. F. Eden. "Left hemisphere specialization for the control of voluntary movement rate." Neuroimage, vol. 22, no. 1, pp.289-303, 2004.

[6] Chan, C.K. Raymond, J. Huang, and X. Di. "Dexterous movement complexity and cerebellar activation: A meta-analysis." Brain Research Reviews, vol. 59, no. 2, pp. 316-323, 2009.

[7] S. Lee, and Y. Sankai, "Power assist control for walking aid with HAL-3 based on EMG and impedance adjustment around knee joint." Intelligent Robots and Systems, vol. 2, pp.1499. 1504, 2002.

[8] Scholkmann F, Kleiser S, Metz A J, Zimmermann R, Mata Pavia J, Wolf U and Wolf M. "A review on continuous wave functional near-infrared spectroscopy and imaging instrumentation and methodology." Neuroimage, vol. 6, no, 27, pp.85, 2014.

[9] Yamamoto, Toru, and T. Kato. "Paradoxical correlation between signal in functional magnetic resonance imaging and deoxygenated hemoglobin content in capillaries: a new theoretical explanation." Physics in Medicine \& Biology 2.6195, pp.938-938, 1979.

[10]Chen, W.G., et al., "Ischemic and bleeding disease monitoring with fNIRS imager." One case report, 
in Saratov Fall Meeting '98: Light Scattering Technologies for Mechanics, Biomedicine, and Material Science, V.V. Tuchin, V.P. Ryabukho, and D.A. Zimnyakov, Editors. Spie-Int Soc Optical Engineering: Bellingham. pp. 540-544, 1999.

[11]Kwee, I.L. and T. Nakada, "Dorsolateral prefrontal lobe activation declines significantly with age Functional NIRS study." Journal of Neurology, vol. 250, no. 5, pp. 525-529. 2003.

[12]Hoshi, Y., S.J. Chen, and M. Tamura, "Spatiotemporal imaging of human brain activity by functional near-infrared spectroscopy." American Laboratory, vol. 33, no. 20, pp. 35, 2001.

[13] Schroeter, M.L., et al., "Towards a standard analysis for functional near-infrared imaging." Neuroimage, vol. 21, no. 1, pp. 283-290, 2004.

[14]Blumen HM, Holtzer R, Brown LL, Gazes Y, Verghese J, Behavioral and neural correlates of imagined walking and walking-while-talking in the elderly. Hum Brain Mapp, 2014.

[15] Pietro Caliandro, Mariano Serrao, Luca Padua, Gabriella Silvestri, Chiara Iacovelli, Chiara Simbolotti , Silvia Mari, Giuseppe Reale, Carlo Casali and Paolo M. Rossini," Prefrontal cortex as a compensatory network in ataxic gait: A correlation study between cortical activity and gait parameters." Restorative Neurology and Neuroscience, vol. 33, pp. 177-187, 2015.

[16]Lukas Jaeger, Laura Marchal-Crespo, Peter Wolf, Andreas R. Luft, Robert Riener, Lars Michels, Spyros Kollias," On the Modulation of Brain Activation During Simulated Weight Bearing in Supine Gait-Like Stepping." Brain Topogr, vol. 29, pp. 193-205, 2016.

[17] Shimadzu Corporation, Kyoto, Japan, Instruction manual for functional optical imaging system FOIRE-3000 series, Manual No : M587-E005, Rev. B, Oct. 2010.

[18] Picard, Nathalie, and P. L. Strick. "Imaging the premotor areas." Current Opinion in Neurobiology, vol. 11, no. 6, pp.663-672, 2001.

[19]Zengyong, Li, "Wavelet analysis of skin blood oscillations in persons with spinal cord injury and able-bodied subjects." Archives of Physical Medicine \& Rehabilitation, vol. 87, no. 11, pp.7-8, 2007.

[20] Dario Farina, Omar Feix do Nascimento, MarieFranc, oise Lucas, Christian Doncarli. "Optimization of wavelets for classification of movement-related cortical potentials generated by variation of force-related parameters." Journal of Neuroscience Methods, vol. 162, pp.357-363, 2007.

[21] Abry P, Aldroubi A. "Designing multiresolution analysis-type wavelets and their fast algorithms." J Fourier Anal Appl, vol. 2, pp. 135-159, 1995.

[22] Aldroubi A, Abry P, Unser M. "Construction of biorthogonal wavelets starting from any two resolutions." IEEE Trans Sig Proc, vol. 46, pp. 1130-1133, 1998.
[23] V.N. Vapnik, "An overview of statistical learning theory." IEEE Transactions on Neural Networks, vol. 10, no. 10, pp.988-99, 1999. 